\title{
Automorphisms and Distinguishing Numbers of Geometric Cliques
}

\author{
Michael O. Albertson • Debra L. Boutin
}

Received: 2 October 2006 / Revised: 20 August 2007 /

Published online: 15 February 2008

(C) Springer Science+Business Media, LLC 2008

\begin{abstract}
A geometric automorphism is an automorphism of a geometric graph that preserves crossings and noncrossings of edges. We prove two theorems constraining the action of a geometric automorphism on the boundary of the convex hull of a geometric clique. First, any geometric automorphism that fixes the boundary of the convex hull fixes the entire clique. Second, if the boundary of the convex hull contains at least four vertices, then it is invariant under every geometric automorphism. We use these results, and the theory of determining sets, to prove that every geometric $n$-clique in which $n \geq 7$ and the boundary of the convex hull contains at least four vertices is 2-distinguishable.
\end{abstract}

Keywords Geometric automorphism · Geometric clique - Distinguishing number · Determining set $\cdot$ Convex hull boundary

\section{Introduction}

The distinguishing number of a graph $G$ is the smallest integer $d$ so that each vertex of $G$ can be labeled with an integer from $\{1, \ldots, d\}$ in such a way that no automorphism of $G$, other than the identity, preserves the labels. A subset $S$ of vertices of a graph $G$ is called a determining set if whenever two automorphisms agree on the elements of $S$, they agree on all of $G$. That is, the image of $S$ under an arbitrary automorphism determines the automorphism completely. It is straightforward to extend both distinguishing and determining to geometric graphs.

M.O. Albertson (凶)

Department of Mathematics and Statistics, Smith College, Northampton, MA 01063, USA

e-mail: albertson@math.smith.edu

D.L. Boutin ( $\bowtie)$

Department of Mathematics, Hamilton College, Clinton, NY 13323, USA

e-mail: dboutin@hamilton.edu 
Determining sets provide a useful tool for finding distinguishing numbers. To prove that $G$ has distinguishing number of at most 2, it suffices to find a determining set that can be 1-distinguished (as a subset). This method was introduced in [3] where it was used to find the distinguishing numbers of all Kneser graphs. Here, we use this method to show that certain geometric cliques are 2-distinguishable.

The paper is organized as follows. Section 2 gives background on geometric graphs and their automorphisms. It formalizes distinguishing numbers, determining numbers, and the connections between them. It concludes by describing our prior knowledge on distinguishing geometric cliques. Section 3 considers how a geometric automorphism might alter the boundary of the convex hull of a geometric clique. The two theorems proved in this section are of general interest. The first shows that in a geometric clique, the pointwise stabilizer of the vertices on the boundary of the convex hull is the identity. The second shows that in a geometric clique, if the boundary of the convex hull contains at least four vertices, then it is invariant under any geometric automorphism. We give examples to show that this invariance does not necessarily hold when the boundary contains exactly three vertices. Section 4 shows that every geometric clique that has at least seven vertices and a convex hull with at least four vertices on its boundary is 2-distinguishable.

\section{Background}

Given an abstract graph $G$, let $\bar{G}$ denote a geometric graph that realizes $G$. In particular, $V(\bar{G})=\left\{x_{1}, x_{2}, \ldots, x_{n}\right\}$ is a set of $n$ points in general position in $\mathbb{R}^{2}$ and the edge $x_{i} x_{j} \in E(\bar{G})$ is the straight line segment joining the corresponding pair of points. Two edges, say $u v$ and $x y$, are said to cross if the interiors of the line segments from $u$ to $v$ and $x$ to $y$ have nonempty intersection. This occurs precisely when the vertices $u, x, v$, and $y$ are (in cyclic order) the vertices of a convex quadrilateral. See $[13,14]$ for background on geometric graphs. Two geometric graphs, say $\bar{G}$ and $\bar{H}$, are said to be geometrically isomorphic if there exists $\phi$, a graph isomorphism from $G$ to $H$, such that edges $\phi(u) \phi(v)$ and $\phi(x) \phi(y)$ cross in $\bar{H}$ if and only if edges $u v$ and $x y$ cross in $\bar{G}$. A geometric isomorphism from $\bar{G}$ to itself is called a geometric automorphism. Let $\operatorname{Aut}(\bar{G})$ denote the group of geometric automorphisms of $\bar{G}$. It is immediate that if $\bar{G}$ is a geometric realization of $G$, then $\operatorname{Aut}(\bar{G})$ is a subgroup of $\operatorname{Aut}(G)$. We say that subgraph $\bar{G}_{2}$ is a geometric image of subgraph $\bar{G}_{1}$ if there exists $\sigma \in \operatorname{Aut}(\bar{G})$ such that $\sigma\left(\bar{G}_{1}\right)=\bar{G}_{2}$. We call $\bar{K}_{n}$ a geometric clique. The boundary of the convex hull of a geometric clique is important for our investigations: we denote this boundary by $C$. Sometimes we think of $C$ as the polygonal boundary, and sometimes we think of $C$ as the vertex set of that polygon. In the latter context set $c=|C|$.

A labeling $f: V(\bar{G}) \rightarrow\{1, \ldots, d\}$ is said to be $d$-distinguishing if $\phi \in \operatorname{Aut}(\bar{G})$ and $f(\phi(x))=f(x)$ for all $x \in V(\bar{G})$ implies that $\phi=i d$. The distinguishing number of $\bar{G}$, denoted here by $\operatorname{Dist}(\bar{G})$, is the minimum $d$ such that $\bar{G}$ has a $d$-distinguishing labeling. Note that we have a $d$-distinguishing labeling of a geometric graph $\bar{G}$ if and only if the labeling together with the structure of $\bar{G}$ uniquely identifies every vertex. Every geometric graph has a distinguishing labeling since each vertex can be assigned a distinct label. Furthermore, there are geometric graphs, e.g. $\bar{K}_{3}$ or $\bar{K}_{1, n}$, 
for which such a labeling is optimal or nearly optimal. The distinguishing number was introduced in [4] and has seen substantial recent interest [1-3, 5, 7-12, 15].

As an example consider $C_{n}$, the cycle with $n$ vertices. When $3 \leq n \leq 5$, $\operatorname{Dist}\left(C_{n}\right)=3$, but when $n \geq 6$, $\operatorname{Dist}\left(C_{n}\right)=2$. To see this latter claim, consecutively label the vertices of $C_{n}(n \geq 6)$ with $1,1,2,1,2, \ldots, 2$. This will be useful in Sect. 4.

A subset $S \subseteq V(\bar{G})$ is said to be a determining set if whenever $g, h \in \operatorname{Aut}(\bar{G})$ and $g(s)=h(s)$ for all $s \in S$, then $g=h$. The determining number of $\bar{G}$, denoted here by $\operatorname{Det}(\bar{G})$, is the minimum $r$ such that $\bar{G}$ has a determining set of cardinality $r$. Every graph has a determining set, since any set containing all but one vertex is determining. There are geometric graphs, e.g. $\bar{K}_{3}$ and $\bar{K}_{1, n}$, for which such a determining set is optimal or nearly optimal. Determining sets and the determining number were introduced in [6] and were first used for distinguishing in [3].

Every convex $\bar{C}_{n}$ has a determining set consisting of any two nonantipodal vertices. Note that if $n \geq 6$ and $\bar{C}_{n}$ is convex, then $\operatorname{Dist}\left(\bar{C}_{n}\right)=\operatorname{Det}\left(\bar{C}_{n}\right)=2$, but for $3 \leq n \leq 5$, Dist $\left(\bar{C}_{n}\right)=3$ while $\operatorname{Det}\left(\bar{C}_{n}\right)=2$. To illustrate that these numbers depend on the particular geometric realization, note that if $\bar{C}_{4}$ has a crossing, then $\operatorname{Dist}\left(\bar{C}_{4}\right)=2$ while $\operatorname{Det}\left(\bar{C}_{4}\right)=1$.

Vertex stabilizers can be used to characterize determining sets as seen below. If $s \in$ $V(\bar{G})$, let $\operatorname{Stab}(s)$ denote the subgroup of $\operatorname{Aut}(\bar{G})$ that fixes $s$. Recall that $\operatorname{Stab}(S)=$ $\bigcap_{s \in S} \operatorname{Stab}(s)$ is the pointwise stabilizer of the set $S$.

Theorem 1 [6] Let $S$ be a subset of the vertices of the graph $\bar{G}$. Then $S$ is a determining set for $\bar{G}$ if and only if $\operatorname{Stab}(S)=\{i d\}$.

In order to connect determining sets and distinguishing numbers we need to define what it means to $d$-distinguish a subset of $V(\bar{G})$. Let $S \subseteq V(\bar{G})$. A labeling $f: S \rightarrow$ $\{1, \ldots, d\}$ is called $d$-distinguishing if $\phi \in \operatorname{Aut}(\bar{G})$ and $f(\phi(x))=f(x)$ for all $x \in S$ implies that $\phi \in \operatorname{Stab}(S)$. Note that such a $\phi$ fixes $S$ pointwise though it might move vertices that are not in $S$.

Theorem 2 [3] A (geometric) graph can be d-distinguished if and only if it has a determining set that is $(d-1)$-distinguishable.

Distinguishing geometric graphs was first considered in [2]. It is straightforward to see that when $\bar{K}_{n}$ is convex, $\operatorname{Dist}\left(\bar{K}_{n}\right)=3$ when $3 \leq n \leq 5$, while $\operatorname{Dist}\left(\bar{K}_{n}\right)=2$ when $n \geq 6$. It was shown there that for $n \geq 6$, there exists a rigid $\bar{K}_{n}$. More significantly it was shown that, with the exception of the nonconvex $\bar{K}_{4}$ whose distinguishing number is 4 , every geometric clique can be 3 -distinguished. We also made the following conjecture.

Conjecture 1 [2] If $n \geq 7$, then $\operatorname{Dist}\left(\bar{K}_{n}\right) \leq 2$.

Figure 1 displays three nonconvex geometric cliques that have distinguishing number 3. The last two geometric cliques show that Conjecture 1 is best possible.

In this paper we use Theorem 2 to prove a weakening of Conjecture 1. Our result requires the boundary of the convex hull to contain at least four vertices. The case when the boundary of the convex hull has exactly three vertices remains open. 
Fig. 1 Three geometric cliques with distinguishing number 3
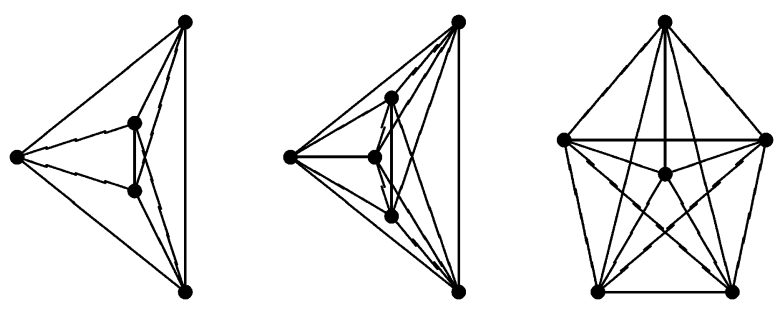

\section{Convex Hulls of Geometric Cliques}

We now present two theorems that describe the effect that a geometric automorphism can have on the boundary of the convex hull of a geometric clique.

Theorem 3 The pointwise stabilizer of the vertices of the boundary of the convex hull of $\bar{K}_{n}$ is trivial.

Proof Let $C=u_{1} u_{2} \cdots u_{c}$ denote the boundary cycle of the convex hull. For each vertex $v$ in the interior of $\bar{K}_{n}$, let $N(v)=\left(N_{1}(v), \ldots, N_{c}(v)\right)$ where $N_{i}(v)$ equals the number of vertices of $\bar{K}_{n}$ interior to the triangle $\Delta v u_{i} u_{i+1}$ with subscript arithmetic modulo $c$. If $v$ and $w$ are two vertices interior to $\bar{K}_{n}$, suppose the ray that begins at $v$ and proceeds through $w$ intersects $C$ in the edge $u_{i} u_{i+1}$. Clearly $\Delta v u_{i} u_{i+1}$ contains $w$ as well as all of the vertices interior to $\Delta w u_{i} u_{i+1}$. Thus $N_{i}(v)>N_{i}(w)$ and consequently all $N(v)$ 's are distinct. A vertex $z$ is interior to $\Delta v u_{i} u_{i+1}$ if and only if for every $j \neq i, i+1$, the edge $z u_{j}$ crosses exactly one of $v u_{i}$ or $v u_{i+1}$. Now suppose $g \in \operatorname{Stab}(C)$. Since this geometric automorphism preserves crossings, $N(g(v))=N(v)$, and thus $g$ must be the identity.

Recall that a set of vertices is said to be invariant if every automorphism maps the set to itself.

Theorem 4 If the boundary of the convex hull of $\bar{K}_{n}$ contains at least four vertices, then it is invariant under every geometric automorphism.

Proof Suppose that the boundary $C$ is not invariant. Then there exists $g \in \operatorname{Aut}\left(\bar{K}_{n}\right)$ so $g(C) \neq C$. Since $g$ is a geometric automorphism, each of $C$ and $g(C)$ is a $c$-cycle of uncrossed edges. Let $\bar{H}$ be the geometric subgraph induced by $C$.

Suppose there exists a vertex $x$ interior to the boundary of the convex hull of $g(\bar{H})$. Let $y$ be a vertex of $V(C)-V(g(C))$. Since $y$ is outside $g(C)$, the edge from $x$ to $y$ must cross $g(C)$-a contradiction. Thus there are no vertices interior to $g(C)$. Next suppose that some edge $e=x y$ not of $g(\bar{H})$ crosses an edge of $g(\bar{H})$. It cannot cross an edge of $g(C)$ since these must be uncrossed, so both $x$ and $y$ must be interior to $g(C)$-a contradiction. Thus the only edges that cross $g(\bar{H})$ are those of $g(\bar{H})$.

The edges of $\bar{H}$ partition the interior of $C$ into regions. Each region is bounded by (parts of) several edges. Suppose two vertices of $V(g(C))-V(C)$ live in different regions. Then $g(C)$ must have consecutive vertices, say $w=g\left(u_{i}\right)$ and $z=g\left(u_{i+1}\right)$, contained in different regions, say $R_{w}$ and $R_{z}$. But then the edge $w z \in g(C)$ must 
Fig. 2 A $\bar{K}_{9}$ with a noninvariant $C$

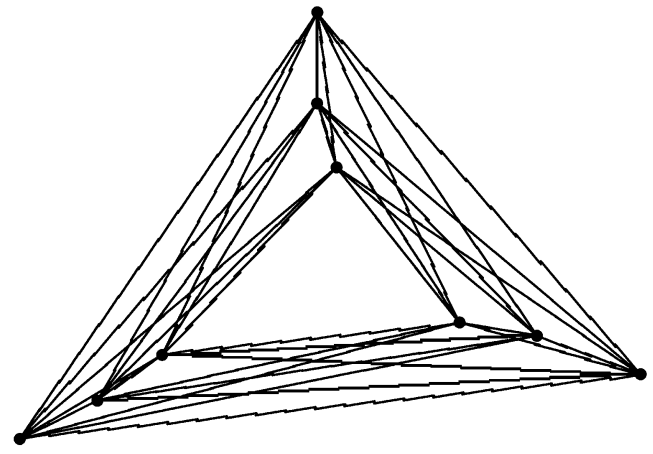

cross a boundary of $R_{w}$. But the edges of $g(C)$ must be uncrossed. Thus the vertices of $V(g(C))-V(C)$ must live in a single region. Call this region $R$.

To arrive at our final contradiction note that, since $c \geq 4$, no region has all vertices of $C$ on its boundary. Then, in particular, there is a vertex $x$ of $V(C)$ that is not on the boundary of $R$. Let $y$ be any vertex of $V(g(C))-V(C)$. Then the edge $y x$ must cross the boundary of $R$ and therefore crosses an edge of $\bar{H}$. Thus $\bar{H}$ has edges which are crossed by edges not of $\bar{H}$. But this is impossible since $\bar{H}$ is a geometric image of $g(\bar{H})$. Thus if $c \geq 4$, there is no $g \in \operatorname{Aut}\left(\bar{K}_{n}\right)$ for which $g(C) \neq C$.

Theorem 4 does not hold when $c=3$. The nonconvex $\bar{K}_{4}$ is an example with distinguishing number 4 . An infinite family of examples with distinguishing number 2 has as its first member a $\bar{K}_{6}$ whose vertices form two nested triangles. The second member of this family is shown in Fig. 2. In each of these examples there is an automorphism that maps the boundary of the convex hull to an interior triangle all of whose edges are uncrossed.

Theorem 5 If a geometric clique has four or more vertices on the boundary of its convex hull, then it has a determining set consisting of any two nonantipodal vertices of this boundary.

The above result follows immediately from the two preceding theorems and the remark concerning the determining set of a convex $\bar{C}_{n}$. The nonconvex $\bar{K}_{4}$ shows that it is best possible.

\section{Distinguishing Geometric Cliques}

Theorem 6 If the boundary of the convex hull of $\bar{K}_{n}$ contains at least six vertices, then $\operatorname{Dist}\left(\bar{K}_{n}\right) \leq 2$.

Proof Theorem 4 implies that $C$ is invariant. The 2-distinguishing labeling of $C_{n}$ given in Sect. 2 fixes $C$. Theorem 3 implies that $\operatorname{Dist}\left(\bar{K}_{n}\right) \leq 2$.

Theorem 7 If the boundary of the convex hull of $\bar{K}_{n}$ contains at least four vertices and the interior contains at least two vertices, then $\operatorname{Dist}\left(\bar{K}_{n}\right) \leq 2$. 
Fig. $3 c \geq 4: n \geq c+2$ : Case $\mathrm{i}$

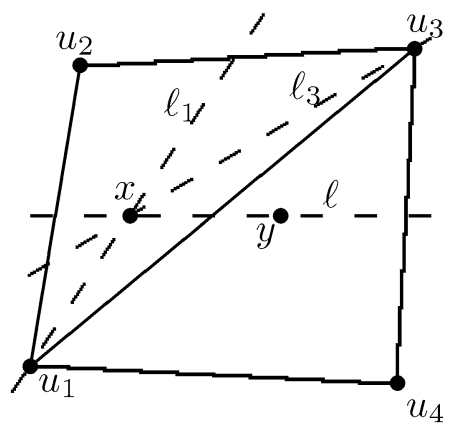

Proof If $x$ and $y$ are vertices interior to $\bar{K}_{n}$, let $\ell$ denote the line in $\mathbb{R}^{2}$ through $x$ and $y$. Since the vertices of $\bar{K}_{n}$ are in general position, $\ell$ intersects $C$ in two points interior to distinct edges in $C$.

Case $i$ The two edges of $C$ that $\ell$ intersects are not incident. Suppose one edge is $u_{1} u_{2}$ while the other edge is $u_{3} u_{4}$. We may assume that $u_{1}$ and $u_{4}$ are in one half plane bounded by $\ell$ while $u_{2}$ and $u_{3}$ are in the other half plane. Since $u_{1}, u_{2}, u_{3}$, $u_{4} \in C, \diamond u_{1} u_{2} u_{3} u_{4}$ is a convex quadrilateral. Note that while the edges $u_{1} u_{2}$ and $u_{3} u_{4}$ are in $C$, the edges $u_{2} u_{3}$ and $u_{4} u_{1}$ might not be in $C$. We may assume that if we rotate a line through the vertex $u_{1}$ so that it sweeps out the convex quadrilateral $\diamond u_{1} u_{2} u_{3} u_{4}$, then this line encounters $x$ before it encounters $y$. We may also assume that $x$ is in $\Delta u_{1} u_{2} u_{3}$. Should either of these not occur, renaming will suffice to make it so.

Let $\ell_{1}\left(\right.$ resp. $\left.\ell_{3}\right)$ denote the line through $u_{1}$ (resp. $u_{3}$ ) and $x$. Since $x$ is inside $\triangle u_{1} u_{2} u_{3}, \diamond u_{1} x u_{3} u_{4}$ is a convex quadrilateral. By assumption $y$ is in the half plane bounded by $\ell_{1}$ that contains $u_{3}$ and $u_{4}$. Furthermore, since $\ell$ intersects the edge $u_{3} u_{4}$, $y$ must be in the half plane bounded by $\ell_{3}$ that contains $u_{4}$. Consequently $y$ is in the interior of $\diamond u_{1} x u_{3} u_{4}$ (see Fig. 3).

Let $\bar{H}$ denote the subgraph of $\bar{K}_{n}$ induced by $T=\left\{u_{1}, x, u_{3}, u_{4}, y\right\}$. Since $T$ contains three vertices of $C$, at least one pair of these are not antipodal in $C$. By Theorem 5, $T$ is a determining set. It remains to show that $T$ is 1-distinguishable.

Suppose that we label each vertex of $T$ with 1 and leave the vertices not in $T$ unlabeled. Now suppose $\sigma \in \operatorname{Aut}\left(\bar{K}_{n}\right)$ preserves labels. This is equivalent to supposing that $T$ is invariant under some $\sigma \in \operatorname{Aut}\left(\bar{K}_{n}\right)$. Then $\sigma$ induces an automorphism on $\bar{H}$. Since the convex hull of $\bar{H}$ contains four vertices, by Theorem 4 it is invariant under geometric automorphism. Thus $\sigma$ sends $\left\{u_{1}, x, u_{2}, u_{3}\right\}$ to itself. Thus $\sigma$ fixes $y$. Furthermore, since $T \cap V(C)=\left\{u_{1}, u_{2}, u_{3}\right\}$, and both these sets are invariant under geometric automorphism, $\sigma$ must map $\left\{u_{1}, u_{3}, u_{4}\right\}$ to itself. Thus $\sigma$ must also fix $x$. Within $\bar{H}$, the edges $u_{1} x$ and $u_{3} x$ are uncrossed, while the edge $u_{4} x$ is crossed by the edge $u_{1} u_{3}$. Thus the only nontrivial possibility for $\sigma$ must switch $u_{1}$ and $u_{3}$. But since, within $\bar{H}$, exactly one of $y u_{1}$ and $y u_{3}$ will be uncrossed, no such automorphism exists. Thus $\sigma=i d \in \operatorname{Stab}(H)$. Therefore $T$ is a 1-distinguishable subset of $V\left(\bar{K}_{n}\right)$.

Case $i i$ The two edges of $C$ that $\ell$ intersects are incident. Suppose these two edges are $u_{1} u_{2}$ and $u_{2} u_{3}$. We may assume that if we rotate a line through the vertex $u_{2}$ so that it sweeps out the sector from $u_{1}$ to $u_{3}$ that it encounters $x$ before it encounters $y$. Let $\ell_{2}$ denote the line determined by $u_{2}$ and $x$. Since $c \geq 4$ there is a vertex 
Fig. $4 \quad c \geq 4: n \geq c+2$ : Case ii

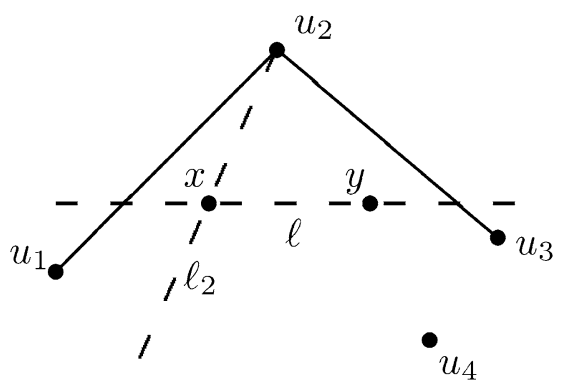

$u_{4} \in C$ such that $u_{4}$ is in the half plane determined by $\ell$ that contains both $u_{1}$ and $u_{3}$. If $u_{4}$ is in the half plane determined by $\ell_{2}$ that contains $u_{1}$, let $\bar{H}$ denote the subgraph of $\bar{K}_{n}$ induced by $T=\left\{u_{1}, u_{2}, u_{4}, x, y\right\}$. Alternatively, if $u_{4}$ is in the half plane determined by $\ell_{2}$ that contains $u_{3}$, let $\bar{H}$ denote the subgraph of $\bar{K}_{n}$ induced by $T=\left\{u_{2}, u_{3}, u_{4}, x, y\right\}$. This latter alternative is shown in Fig. 4. In either situation, the proof can be completed as in Case $i$.

The following corollary is an immediate consequence of the two preceding theorems.

Corollary 1 If the boundary of the convex hull of $\bar{K}_{n}$ contains at least four vertices and $n \geq 7$, then $\operatorname{Dist}\left(\bar{K}_{n}\right) \leq 2$.

Acknowledgement The authors thank an anonymous referee for suggestions that shortened and clarified several of the proofs in this paper.

\section{References}

1. Albertson, M.O.: Distinguishing Cartesian powers of graphs. Electron. J. Comb. 12 (2005). Note 17 (Electronic)

2. Albertson, M.O., Boutin, D.L.: Distinguishing geometric graphs. J. Graph. Theory 53, 135-150 (2006)

3. Albertson, M.O., Boutin, D.L.: Using determining sets to distinguish Kneser graphs. Electron. J. Comb. 14(1) (2007). Research Paper 20 (Electronic)

4. Albertson, M.O., Collins, K.L.: Symmetry breaking in graphs. Electron. J. Comb. 3(1) (1996). Research Paper 18 (Electronic)

5. Bogstad, B., Cowen, L.J.: The distinguishing number of the hypercube. Discrete Math. 283(1-3), 29-35 (2004)

6. Boutin, D.L.: Identifying graph automorphisms using determining sets. Electron. J. Comb. 13(1) (2006). Research Paper 78 (Electronic)

7. Chan, M.: The distinguishing number of the direct product and the wreath product action. J. Algebr. Comb. 24, 331-335 (2006)

8. Chan, M.: The maximum distinguishing number of a group. Electron. J. Comb. 13 (2006). Research Paper 70 (Electronic)

9. Collins, K.L., Trenk, A.N.: The distinguishing chromatic number. Electron. J. Comb. 13(1) (2006). Research Paper 16 (Electronic)

10. Imrich, W., Klavzar, S.: Distinguishing Cartesian powers of graphs. J. Graph. Theory 53(3), 250-260 (2006)

11. Klavžar, S., Wong, T.-L., Zhu, X.: Distinguishing labellings of group action on vector spaces and graphs. J. Algebra 303(2), 626-641 (2006) 
12. Klavzar, S., Zhu, X.: Cartesian powers of graphs can be distinguished with two labels. Eur. J. Comb. 28(1), 303-310 (2007)

13. Pach, J.: Finite point configurations. In: Handbook of Discrete and Computational Geometry, 2nd edn. Discrete Math. Appl., pp. 219-238. CRC, Boca Raton (2004)

14. Pach, J. (ed.): Towards a Theory of Geometric Graphs. Contemporary Mathematics, vol. 342. American Mathematical Society, Providence (2004)

15. Tymoczko, J.: Distinguishing numbers for graphs and groups. Electron. J. Comb. 11(1) (2004). Research Paper 63 (Electronic) 